REVISTA DE URBANISMO

№21, diciembre 2009

ISSN 0717-5051

\title{
Un Plan Regulador para Cartagena, una ciudad de la costa central de Chile: una visión de futuro en 1976
}

\section{A master plan for Cartagena, a city on the central coast of Chile: a vision in 1976}

\author{
Pávez Reyes, M. I sabel \\ Doctora en Arquitectura y Urbanismo, investigadora en la Facultad de \\ Arquitectura y Urbanismo de la Universidad de Chile. \\ Palabras Claves: TPLANIFICACIÓN URBANA Y REGIONAL, PLANIFICACIÓN \\ URBANA DE LA DÉCADA LOA ñoa 1970S, JUAN PARROCHIA BEGUIN, MAURICIO \\ MUÑOZ ROJ AS, VICENTE GÁMEZ BASTÉN, DEPUR - FAU- U. DE CHILE. \\ URBAN AND REGI ONAL PLANNING, URBAN PLANNING IN THE DECADE OF THE \\ 1970S, JUAN PARROCHIA BEGUIN, MAURICIO MUNOZ ROJAS, VICENTE GÁMEZ \\ BASTÉN, DEPUR - FAU - U. CHILE.

\section{Citación:} \\ Pávez Reyes, M. Isabel. URBAN AND REGI ONAL PLANNING, URBAN PLANNI NG \\ IN THE DECADE OF THE 1970S, JUAN PARROCHIA BEGUIN, MAURICIO MUNOZ \\ ROJ AS, VICENTE GÁMEZ BASTÉN, DEPUR - FAU - U. CHILE. En: Revista de \\ Urbanismo, $N^{\circ} 21$, Santiago de Chile, publicación electrónica editada por el \\ Departamento de Urbanismo, F.A.U. de la Universidad de Chile, junio de 2008, \\ I.S.S.N. 0717-5051.
}

\section{Resumen}

Se presenta la reseña de un proyecto de planificación urbana, conforme a la escuela desarrollada por la FAU de la Universidad de Chile, durante la etapa madura del Estado Planificador Urbano-Regional chileno.

\section{Abstract}

IT provides an overview of an urban planning project, developed under the FAU School of the University of Chile, during the mature stage of the Urban-Regional Planning State of Chile.

Un Proyecto de Plan Regulador para la Comuna de Cartagena -V Región de Chile, realizado en el Departamento de Planificación Urbano-Regional de la Facultad de Arquitectura y Urbanismo de la Universidad de Chile en 1976-, imaginó el balneario de Cartagena en la perspectiva de las necesidades de entonces futuras del desarrollo del 
borde costero central de Chile.

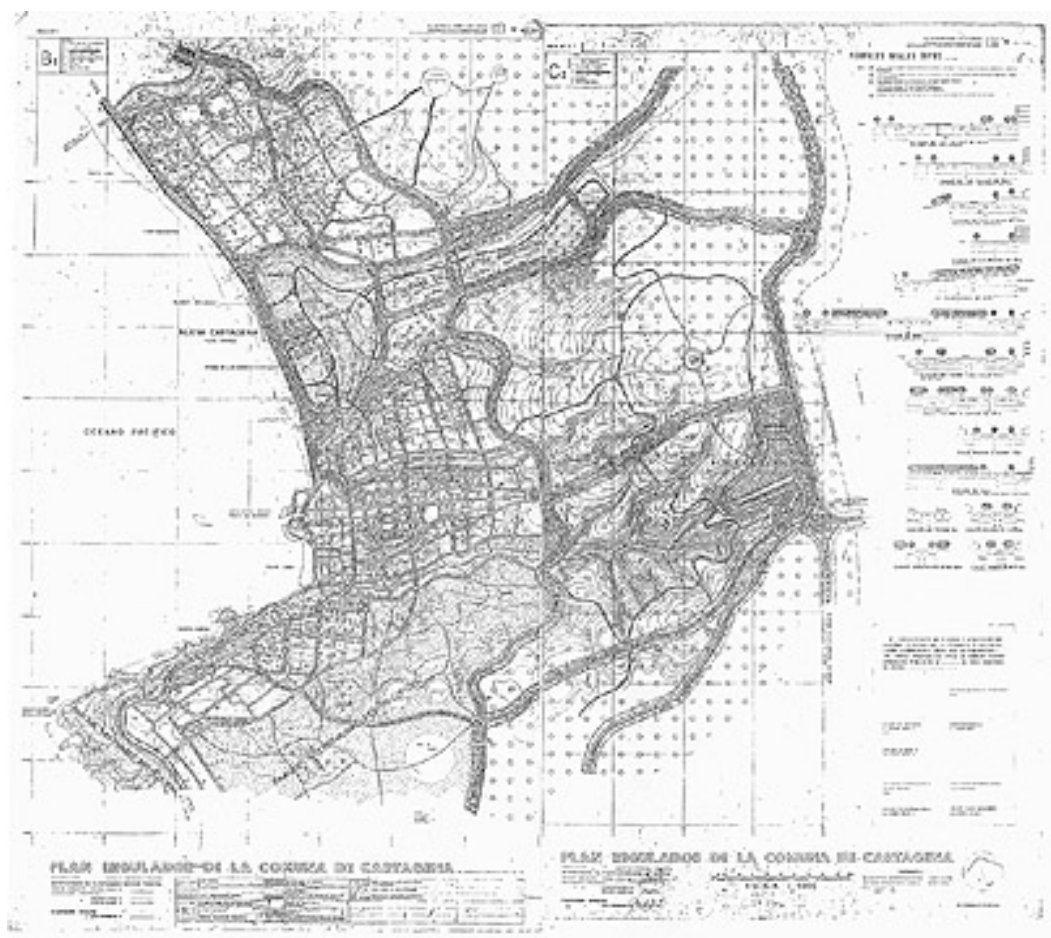

Fig. 1. Proyecto de Plan Regulador de la Comuna de Cartagena 1976, DEPUR, FAU, U. de Chile no realizado.

Fte.: Copia matriz en dos láminas, transparente, escala 1:50.000, en Archivo del D. de Urbanismo FAU, U. de Chile.

Estudió cuidadosamente los espacios públicos viales y verdes de Cartagena, aumentando la conexidad y conectividad de la comuna y ciudad-, y enriqueciendo y diversificando los espacios verdes en todas las escalas. 


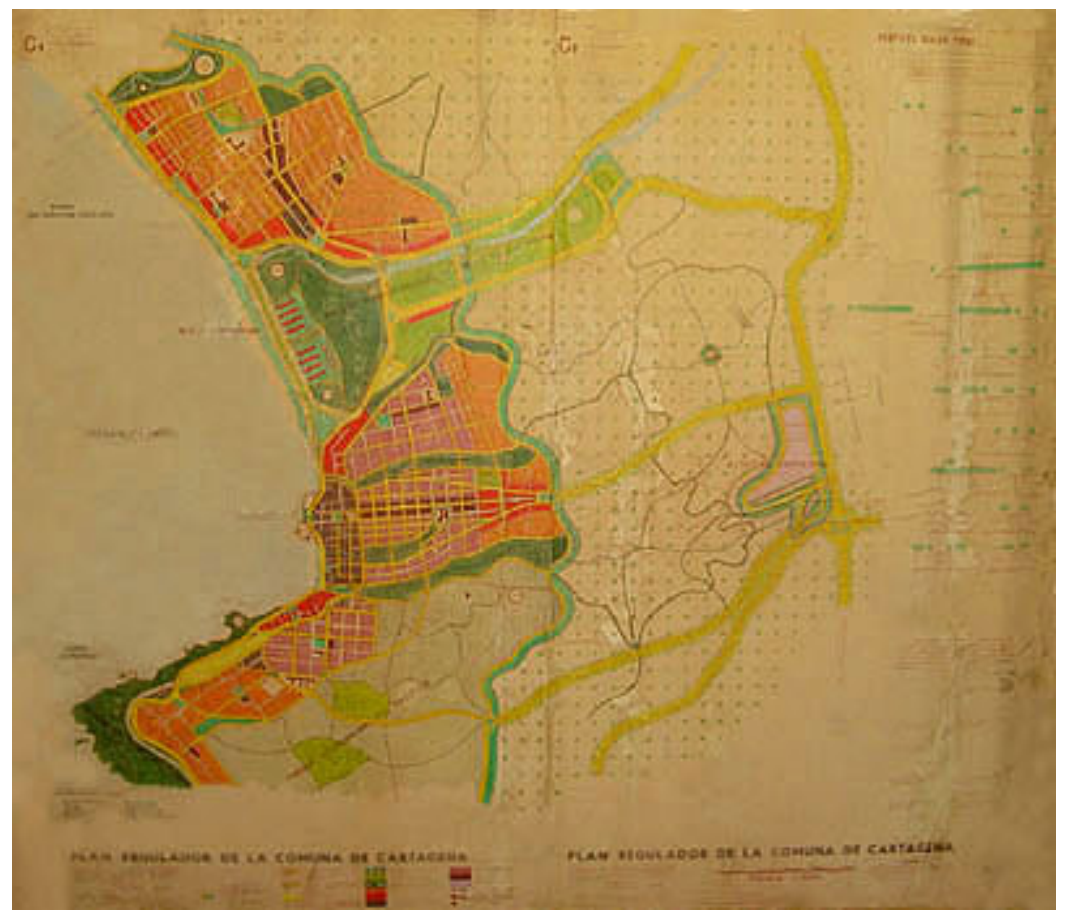

Fig. 2. Proyecto de Plan Regulador de la Comuna de Cartagena 1976, DEPUR, FAU, U. de Chile no realizado.

Fte.: Copia coloreada a mano, en dos láminas, escala 1:5.000, en Biblioteca Central Sección Mapoteca, FAU, U. de Chille.

Destaca el trazado de una "Avda. Panorámica" en la cota 100, con un perfil asimétrico de $80 \mathrm{~m}$. El polígono urbano se conecta a un "Camino Intercomunal Exterior" ubicado aproximadamente en la cota $200 \mathrm{~m}$, por los caminos "del Valle", del "Cerro", de "Cartagena". Un camino "del Centinela" converge, junto al "Camino de San Antonio", hacia una "Micro Zona Industrial Exclusiva", y algunos equipamientos turísticos ubicados en los altos de Cartagena y junto a un nudo vial para conexión tanto a Santiago como al Parque Microregional de los cerros de Cartagena (Miltín).

Un "Camino Panorámico de un perfil entre 15 y 20 m en la cota 150, esto es entre la ciudad y la antes citada "Avenida Panorámica", unía diversos miradores de interés turístico.

Junto al mar, propuso una costanera de carácter intercomunal, el "Camino de las Playas", con un perfil de ancho variable entre 30 y 100 m, incluyendo rambla y el "Paseo de las Dunas" en la zona llamada Nueva Cartagena.

Propuso parques de barrio, como el "Parque Las Cigueñas" -incluyendo un Museo Folklórico- en el límite con la comuna de El Tabo; un "Parque Microregional del Valle de Cartagena", estructurado a partir del estero de Cartagena, teniendo como remate las dunas de Cartagena, incluyendo un Acuario y un Casino en su polígono. 


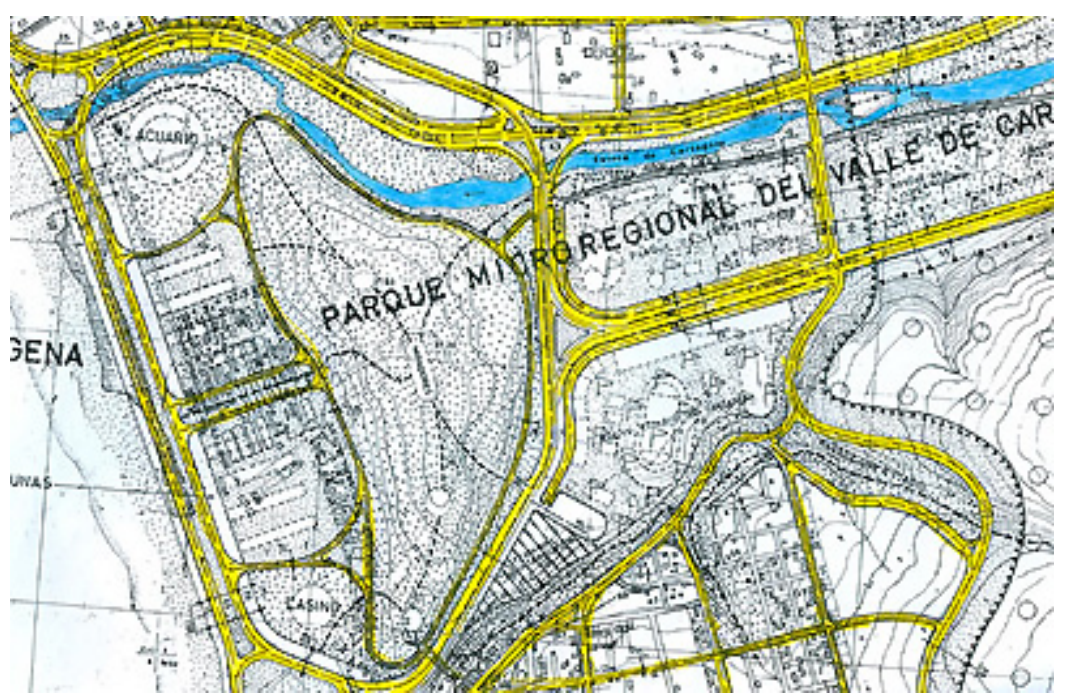

Fig.3. Proyecto de Plan Regulador de la Comuna de Cartagena 1976, DEPUR, FAU, U. de Chile no realizado. Detalle de Zona "Parque Micro Regional del Valle de Cartagena", rematando en el "Camino de las Playas" y el "Paseo de las Dunas".

Fte.: Copia coloreada a mano por R. de Urbanismo, escala 1:5.000, en Biblioteca Central Sección Mapoteca, FAU, U. de Chile.

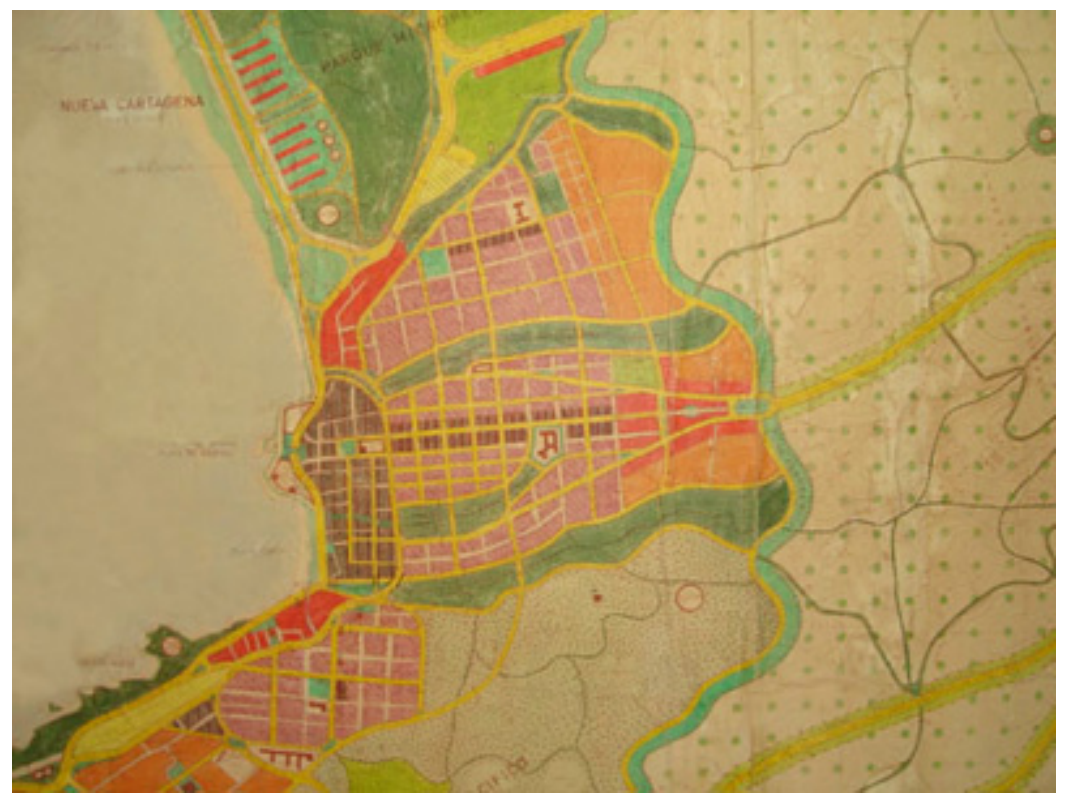

Fig.4. Proyecto de Plan Regulador de la Comuna de Cartagena 1976, DEPUR, FAU, U. de Chile no realizado. Detalle de zona céntrica.

Fte.: Copia coloreada a mano, en dos láminas, escala 1:5.000, en Biblioteca Central Sección Mapoteca, FAU, U. de Chile.

Los parques "El Tranque", "Las Mercedes y "Miraflores" se propusieron en las quebradas de la zona centro de la ciudad. Un "Parque Santuario del Litoral", se dispuso compartido con la comuna de San Antonio, y el "Parque Intercomunal del Pacífico", en la zona alta al sur de la ciudad, incluyendo equipamientos deportivos y la tumba del poeta Vicente Huidobro. 


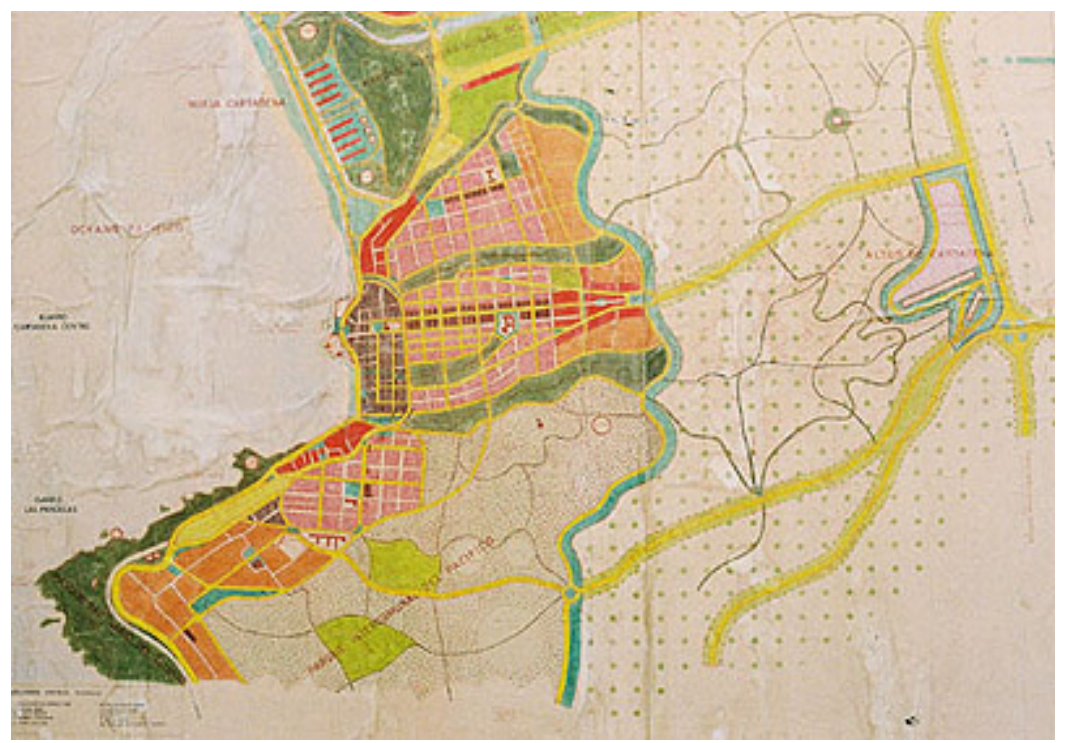

Fig. 5. "Parque intercomunal del Pacífico" (abajo) y "Micro Zona Industrial Exclusiva" en los altos de Cartagena (der.)

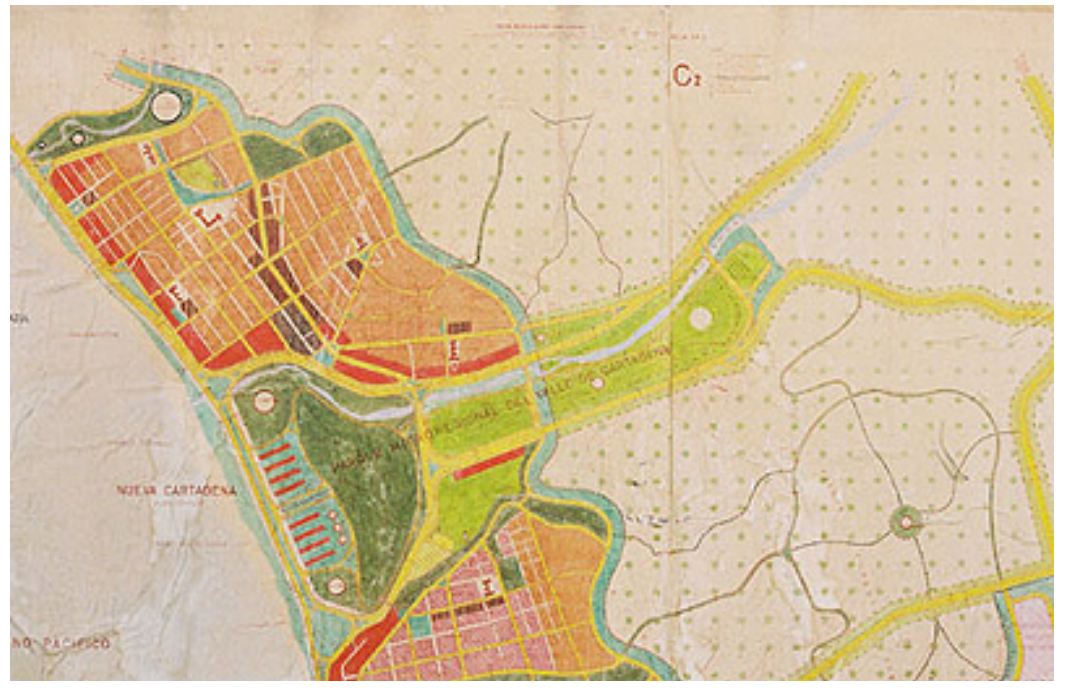

Fig. 6. Zona Norte con Nueva Cartagena. 

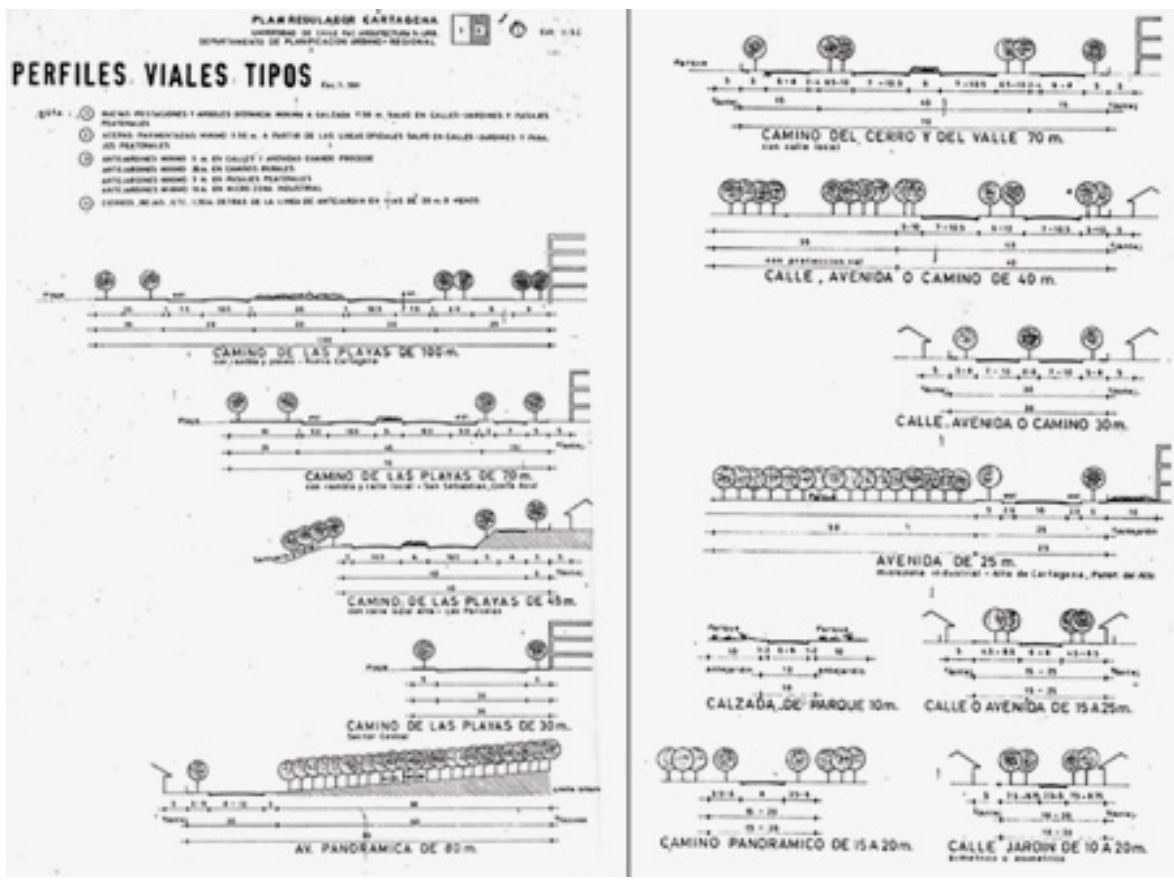

Figs. 7 y 8. Perfiles viales tipos. Proyecto de Plan Regulador de la Comuna de Cartagena 1976, DEPUR, FAU, U. de Chile no realizado.

Fte.: Copia matriz en dos láminas, transparente, escala 1:5.000, en Archivo del D. de Urbanismo FAU, U. de Chile.

Propuso una "Micro Zona Industrial Exclusiva" en los altos de Cartagena, y un terminal de transporte terrestre. Estableció diez monumentos históricos protegidos. Estableció, entre otros usos compatibles, diversos tipos de zonas residenciales, entre 100 y 1.000 hab./ha. El alcance de sus proposiciones de largo plazo, como balneario popular destinado a servir las necesidades del área metropolitana principal de Chile -Santiago- y su región (6 millones de habitantes), no tuvo acogida en el nivel local de la época y fue desestimado sin mayor debate.
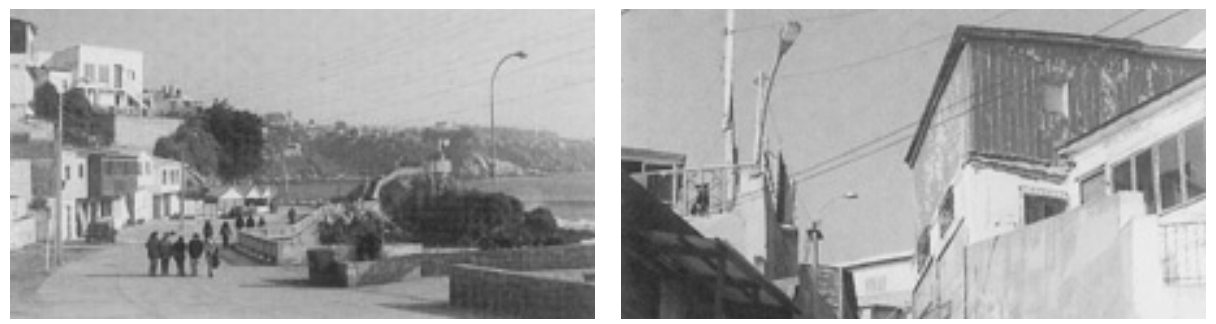


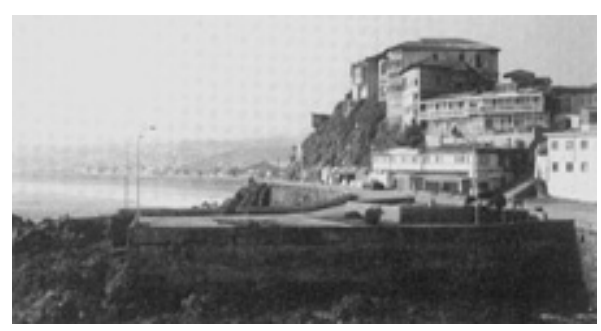

Figs.9, 10 y 11. Vistas de Cartagena. Década de 1990.

Fte.: Archivo D. de Urbanismo, FAU U. de Chile.

El Planificador Consultor fue el Arqto. Juan Parrochia Beguin, y formaron parte del equipo de trabajo de la época los planificadores Arqtos. Mauricio Muñoz Rojas, y Vicente Gámez Bastén. En la etapa de estudio participaron diversos otros investigadores del Departamento de Planificación Urbano-Regional, (planos del polígono urbano a Esc. 1.5000; planos del polígono intercomunal a Esc. 1.50.000).

Actualmente ese balneario recibe más de medio millón de habitantes durante el verano, sin estar preparado para ello, lo cual se traduce en congestión vehicular, ocupación indebida de playas, un cuadro generalizado de problemas de salud pública y conflictos de interacción entre personas visitantes, y entre visitantes y residentes, entre otros.

Revista Electrónica del Departamento de Urbanismo Facultad de Arquitectura y Urbanismo | Universidad de Chile

Fundada en 1999 